\title{
$A b$ initio simulation of laser-induced water decomposition close to carbon nanotubes
}

\author{
Yoshiyuki Miyamoto, ${ }^{1}$ Hong Zhang,,${ }^{2, *}$ Xinlu Cheng, ${ }^{3}$ and Angel Rubio ${ }^{4,5,6}$ \\ ${ }^{1}$ Research Center for Computational Design of Advanced Functional Materials, \\ National Institute of Advanced Industrial Science and Technology (AIST), Central 2, 1-1-1 Umezono, \\ Tsukuba, Ibaraki 305-8568, Japan \\ ${ }^{2}$ College of Physical Science and Technology, Sichuan University, Chengdu 610065, China \\ ${ }^{3}$ Institute of Atomic and Molecular Physics, Sichuan University, Chengdu 610065, China \\ ${ }^{4}$ Max Planck Institute for the Structure and Dynamics of Matter and Center for Free-Electron Laser Science, \\ Luruper Chaussee 149, 22761 Hamburg, Germany \\ ${ }^{5}$ Nano-Bio Spectroscopy Group and ETSF, Universidad del País Vasco, 20018 San Sebastian, Spain \\ ${ }^{6}$ Center for Computational Quantum Physics, Flatiron Institute, 162 Fifth Avenue, New York, New York 10010, USA
}

(Received 7 September 2018; published 22 April 2019)

\begin{abstract}
First-principles simulations were used to investigate water $\left(\mathrm{H}_{2} \mathrm{O}\right)$ decomposition induced by a femtosecond laser with high flux $\sim 1 \times 10^{20}$ photons $/\left(\mathrm{sec} \mathrm{cm}^{2}\right)$. One goal of our research is to find metamaterials that locally enhance the laser field to reduce the threshold laser intensity required to decompose $\mathrm{H}_{2} \mathrm{O}$ molecules. In this work, small-diameter $(6.3 \AA)$ single-walled carbon nanotubes were found to reduce the threshold power by $90 \%$ compared with the power required to decompose $\mathrm{H}_{2} \mathrm{O}$ in the gas phase. The present results suggest a strategy for the design of materials with high energy efficiency for $\mathrm{H}_{2} \mathrm{O}$ decomposition based on polarizability and morphology (curvature) to enhance the local field. We demonstrate that carbon nanotubes enhance the local field resulting in a power enhancement of approximately eight times.
\end{abstract}

DOI: 10.1103/PhysRevB.99.165424

\section{INTRODUCTION}

Photo-induced water $\left(\mathrm{H}_{2} \mathrm{O}\right)$ decomposition can be an alternative to the use of conventional fossil fuels for energy production. The decomposition of $\mathrm{H}_{2} \mathrm{O}$ by light typically requires photons in the vacuum-ultraviolet region [1]. However, the maximum flux of such high-energy photons that can be achieved using currently available light sources is limited. Lower-energy photons at sufficiently high flux may also effectively increase the decomposition yield. Recent developments in femtosecond (fs) laser technology [2-7] allow a high photon flux (high power) to be applied to a focused region of a material to promote nonthermal processes. This high photon flux can be used to promote $\mathrm{H}_{2} \mathrm{O}$ decomposition via multiphoton excitation and attain a high decomposition yield. Herein, based on first-principles simulations, a method for improving the efficiency of photo-induced $\mathrm{H}_{2} \mathrm{O}$ decomposition using nanostructure-based field enhancement is proposed.

In this study, we examined the use of carbon nanotubes (CNTs) as an active optical-field enhancer. The polarizability of CNTs enhances the optical field for $\mathrm{H}_{2} \mathrm{O}$ decomposition in a manner similar to the polarizability of two-dimensional graphitic sheets [8]. However, the source of the polarizability of CNTs is quite different from those in a two-dimensional material and can be derived from two components that arise from parallel and perpendicular polarizations to the nanotube wall [9]. Furthermore, the curvature of CNTs achieves spatial concentration of the optical field. The results of this study

*hongzhang@scu.edu.cn are potentially applicable to the design of materials for laserfield enhancement. Selective syntheses of $(6,5) \mathrm{CNTs}$ with a diameter of $7.5 \AA[10]$ and $(6,4)$ CNTs with a diameter of $6.9 \AA$ [11] were recently reported. In this work, we examined semiconducting $(8,0)$ CNTs with a diameter of $6.3 \AA$ and observed significant enhancement of the optical field. (Metallic CNTs have higher polarizability than semiconducting CNTs, and thus would be expected to show greater enhancements. Therefore, the semiconducting CNTs used here would be expected to provide a lower bound on the optical field enhancement.) Although CNTs are hydrophobic materials, attraction between monolayer $\mathrm{H}_{2} \mathrm{O}$ molecules and CNTs at an average distance of $3.5 \AA$ from the CNT wall have been reported and this attraction originated mainly from van der Waals interactions [12].

By performing real-time electron-ion dynamics based on time-dependent density functional theory (TDDFT) [13] in the presence of fs-laser fields of various intensities, we determined the threshold intensity for $\mathrm{H}_{2} \mathrm{O}$ decomposition. Our simulations demonstrated that CNTs enhance $\mathrm{H}_{2} \mathrm{O}$ decomposition upon fs-laser irradiation with an optical wavelength of $800 \mathrm{~nm}$, allowing the power to be reduced by more than a factor of four compared with the decomposition of an isolated $\mathrm{H}_{2} \mathrm{O}$ molecule in the liquid or gas phase. Furthermore, at an optical wavelength of $400 \mathrm{~nm}$, a power reduction of more than a factor of seven was achieved. The threshold-power reductions obtained using optical wavelengths of 800 and $400 \mathrm{~nm}$ were superior to the reduction obtained using planar sheets [8] by factors of two and three, respectively. Since the considered photon energy exceeds the band gap of $(8,0)$ $\mathrm{CNT}$, the electronic excitation is crucial for optical field enhancement. 


\section{COMPUTATIONAL METHODS}

To investigate the laser-mediated decomposition of $\mathrm{H}_{2} \mathrm{O}$ in the vicinity of an $(8,0) \mathrm{CNT}$, we computed the electron-ion dynamics within the real-time (RT)-TDDFT scheme in the presence of a laser field coupled with classical molecular dynamics (MD) simulation within the scheme of Ehrenfest dynamics [14]. In this work, the optical field was treated using the dipole approximation. The Perdew-Zunger local density approximation (LDA) functional [15] was used. For the laser-mediated decomposition of an isolated $\mathrm{H}_{2} \mathrm{O}$ molecule and an $\mathrm{H}_{2} \mathrm{O}$ molecule above a graphene sheet, the generalized gradient approximation (GGA) with the Perdew-BurkeErnzerhof (PBE) functional [16] afforded quantitatively the same intensity threshold for decomposition as that computed by LDA [8], although these functionals provide different $\mathrm{H}_{2} \mathrm{O}$-graphene van der Waals interactions, resulting in $\mathrm{H}_{2} \mathrm{O}$ graphene distances that differ by $0.45 \AA$. (A detailed discussion of this exchange-correlation functional effect will be provided in a future paper.) Therefore, LDA was employed in all of the present calculations to reduce the computational cost of prolonged simulations. The validity of the Ehrenfest approach has been discussed in Refs. [17,18], and adequate results can be expected in the absence of strong nonadiabatic coupling.

We numerically integrated the time-dependent Kohn-Sham equation,

$$
i \frac{\partial}{\partial t} \psi_{n}^{K S}(\mathbf{r}, t)=\left[H^{K S}(\mathbf{r}, t)+V_{\mathrm{ext}}(\mathbf{r}, t)\right] \psi_{n}^{K S}(\mathbf{r}, t),
$$

by using the fourth-order split-operator scheme $[19,20]$ with a time-step interval of 0.03 a.u. $\left(7.26 \times 10^{-4} \mathrm{fs}\right)$. In Eq. (1), $\psi_{\mathrm{n}}^{K S}(\mathbf{r}, t)$ is the time-dependent Kohn-Sham orbital and $H^{K S}(\mathbf{r}, t)$ is the Kohn-Sham Hamiltonian, which is a functional of the time-dependent charge density $\rho(\mathbf{r}, t)$ consisting of the sum of the norms of the occupied Kohn-Sham orbitals. $V_{\text {ext }}(\mathbf{r}, t)$ incorporates the applied optical electric field (E-field) [21] that interacts with both electrons and ions. A plane-wave basis set was used to express the KohnSham orbitals, charge density, and potentials with a cutoff kinetic energy of $71 \mathrm{Ry}$. The total energy and forces were computed using the momentum-space formalism [22]. This scheme affords a total energy precision of $3 \times 10^{-5} \mathrm{eV}$ per atom and a force precision of $0.05 \mathrm{eV} / \AA$. The interaction between ions and valence electrons was expressed using the norm-conserving pseudopotentials [23] with separable forms [24]. The calculations were performed using the FPSEID code $[25,26]$ and numerical stability was evaluated with the criterion of energy conservation in the presence of a dynamical external field [27].

As a CNT model, semiconducting $(8,0)$ CNTs were used in this study with a triple cell along the CNT axis (96 C atoms per unit cell). The vacuum region was taken in the two orthogonal axes normal to the CNT axis. In one direction, the wall-to-wall distance of the CNT array was taken as $7 \AA$. In the other direction, the wall-to-wall distance was taken as $14 \AA$. Along the longer axis, a time-varying E-field was applied to mimic an optical field, the polarity of which was smoothly inverted in the middle of the vacuum region to satisfy the periodic boundary conditions. In all cases, we used $\Gamma$ points for the

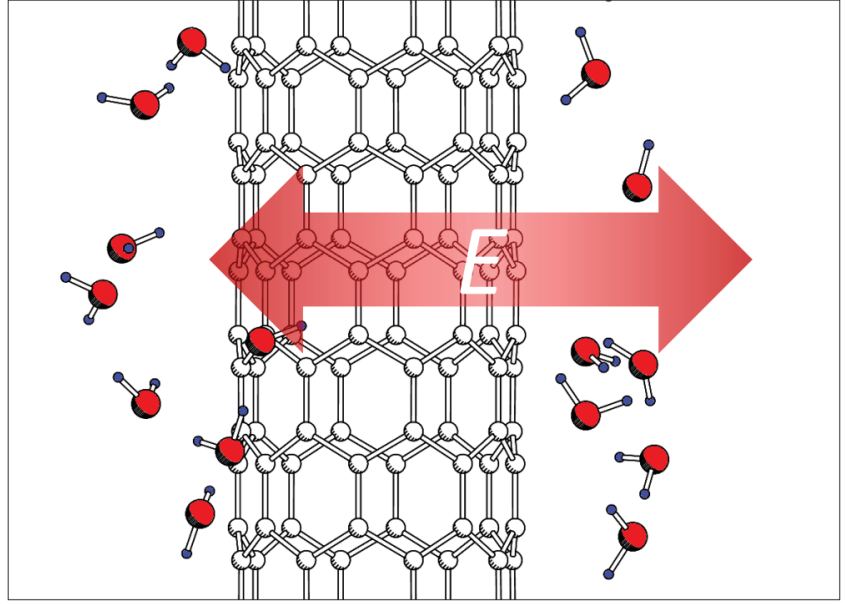

FIG. 1. Structure of an $(8,0)$ CNT surrounded by 15 water molecules. Hatched (red) balls represent $\mathrm{O}$ atoms, white balls represent the $\mathrm{C}$ atoms of the $\mathrm{CNT}$, and the smallest balls represent $\mathrm{H}$ atoms. The red arrow indicates the optical polarization vector. This structure is viewed from a direction normal to the CNT axis.

momentum-space integration [28]. In this work, we focus on the case of a full width at half maximum (FWHM) of $10 \mathrm{fs}$, but larger FWHM values may be investigated in future studies.

\section{RESULTS}

Our simulations revealed that the decomposition of an isolated $\mathrm{H}_{2} \mathrm{O}$ molecule occurred at maximum E-field intensities of 8.5 and $7 \mathrm{~V} / \AA$ for 10 -fs laser pulses with optical wavelengths of 800 and $400 \mathrm{~nm}$, respectively. (For the accessibility of such field intensities, see Ref. [29] and Sec. S1 of the Supplemental Material [30].) We performed RT-TDDFT-MD simulations to examine the $\mathrm{H}_{2} \mathrm{O}$ decomposition with $15 \mathrm{H}_{2} \mathrm{O}$ molecules around an $(8,0) \mathrm{CNT}$; see Fig. 1. Computational details in the MD part of the current simulation are described in Sec. S2 of the Supplemental Material [30].

We set the frequencies of the E-field oscillation corresponding to wavelengths of 800 and $400 \mathrm{~nm}$ with FWHM of 10 fs. The intensity of the E-field was varied from 2 to $7 \mathrm{~V} / \AA$ with an interval of $0.5 \mathrm{~V} / \AA$. Throughout the simulations, we plotted the distances from each $\mathrm{O}$ atom to its first- and secondneighbor $\mathrm{H}$ atoms as a function of time (Fig. 2). We also measured the distance from each $\mathrm{H}$ atom to its first-neighbor $\mathrm{O}$ atoms, the results of which are not shown here. In doing so, it was possible to avoid missing the case in which one $\mathrm{H}(\mathrm{O})$ atom was shared by two $\mathrm{O}$ (three $\mathrm{H}$ ) atoms, resulting in the emission of $\mathrm{H}(\mathrm{O})$ atoms from several $\mathrm{H}_{2} \mathrm{O}$ molecules.

The dynamical change of the $\mathrm{O}-\mathrm{H}$ bond lengths at the threshold intensities of the E-field of $4 \mathrm{~V} / \AA$ for a wavelength of $800 \mathrm{~nm}$ and $2.5 \mathrm{~V} / \AA$ for a wavelength of $400 \mathrm{~nm}$ is shown in Figs. 2(a) and 2(b), respectively. It is interesting to note that significant $\mathrm{O}-\mathrm{H}$ elongation occurred after the optical field had ended. This is consistent with excited-state dynamics initiated by electronic excitation, followed by subsequent lattice dynamics [7]. A corresponding theoretical model for this mechanism was reviewed in Ref. [31]. Compared to the the case of an isolated $\mathrm{H}_{2} \mathrm{O}$ molecule, the threshold intensity 
(a)
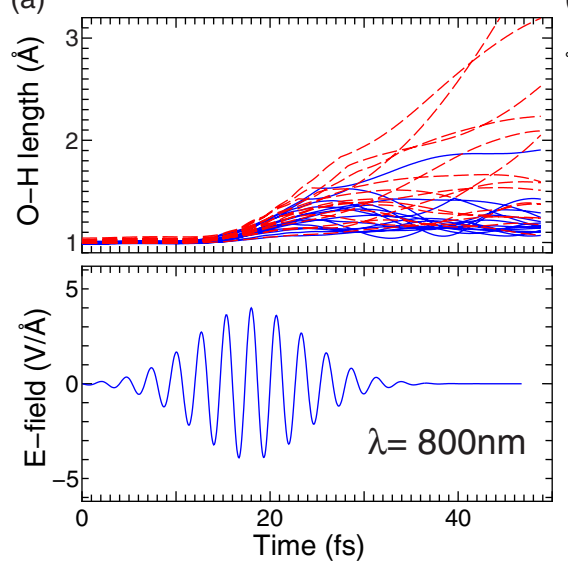

(b)
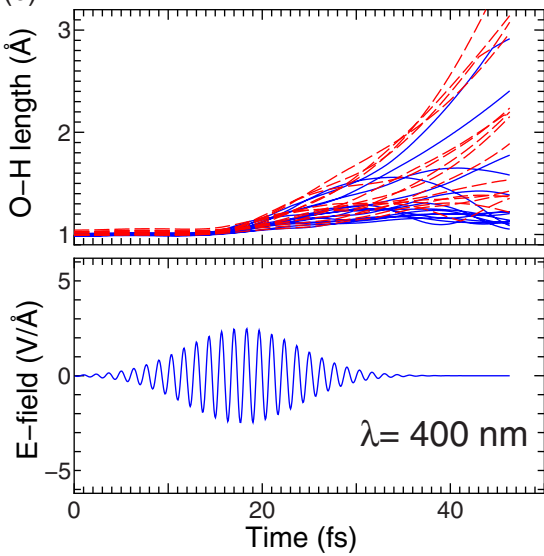

(c)

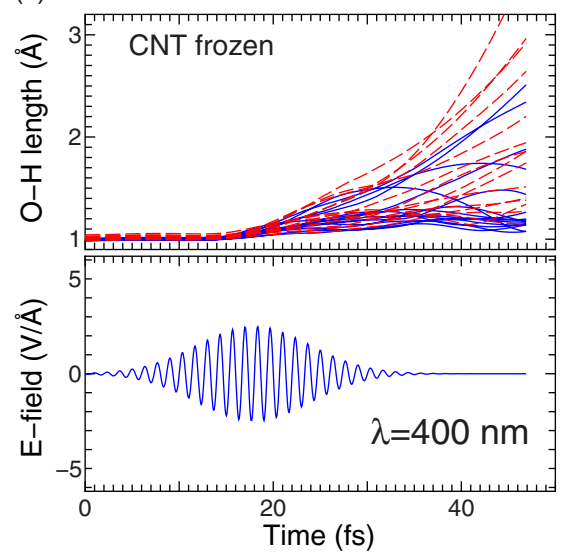

FIG. 2. (a) The top panel depicts the time variation of $\mathrm{O}-\mathrm{H}$ bond lengths. The solid (blue) and dashed (red) curves indicate the first- and second-neighbor $\mathrm{O}-\mathrm{H}$ distances, respectively. The bottom panel depicts the applied optical E-field corresponding to the top panel, with an optical wavelength of $800 \mathrm{~nm}$, an FWHM of $10 \mathrm{fs}$, and a maximum E-field intensity of $4 \mathrm{~V} / \AA$. (b) Same as (a), but for an optical wavelength of $400 \mathrm{~nm}$ and a maximum E-field intensity of $2.5 \mathrm{~V} / \AA$. (c) Same as (b), but CNT is forced to be frozen.

was reduced from 8.5 to $4 \mathrm{~V} / \AA$ at $800 \mathrm{~nm}$, corresponding to a power reduction by a factor of 4.46 . For an optical wavelength of $400 \mathrm{~nm}$, the threshold intensity was reduced from 7 to $2.5 \mathrm{~V} / \AA$, corresponding to a power reduction by a factor of 7.64. (Note that the power is proportional to the square of the optical E-field.) At the threshold intensity and an optical wavelength of $800 \mathrm{~nm}$, as shown in Fig. 2(a), each $\mathrm{O}$ atom has a first-neighbor $\mathrm{H}$ atom at a distance of less than $2 \AA$, whereas some of the $\mathrm{O}$ atoms have second-neighbor $\mathrm{H}$ atoms at a distance of over $3 \AA$. This behavior suggests that $\mathrm{H}_{2} \mathrm{O}$ decomposition generates $\mathrm{H}$ atoms and hydroxyl $(\mathrm{OH})$ groups rather than $\mathrm{O}$ atom radicals. In contrast, at a wavelength of $400 \mathrm{~nm}, \mathrm{O}$ radicals were also emitted, which is denoted by the nearest O-H bond length, shown in Fig. 2(b), increasing over $3 \AA$.

The reduction of the threshold power was attributed to optical E-field enhancement by the CNT. Figure 3(a) shows the time variation of the total E-field (sum of applied and induced E-fields) around a pristine $(8,0)$ CNT for a maximum optical E-field of $3 \mathrm{~V} / \AA$ and a wavelength of $800 \mathrm{~nm}$. The induced field was derived by subtracting the self-consistent potentials on the discrete grids used in the current simulations. The time variation of the induced E-field is plotted at a position $3.3 \AA$ from the CNT wall in the direction of the optical polarization vector. Within the time range studied, the motion of the CNT was not significant; therefore, the time variation of the E-field at a fixed point can be used to discuss the E-field modulation. As shown in Fig. 3(a), the total E-field around the CNT was found to increase with time up to twice the intensity of the applied E-field [32], which is consistent with the reduction in the threshold power for water decomposition.

In contrast, for the wavelength of $400 \mathrm{~nm}$, the field enhancement cannot be simply depicted owing to the significant radial expansion of the CNT due to the weakening of the C-C bonds on the tube wall by electronic excitation. We simulated the $\mathrm{H}_{2} \mathrm{O}$ decomposition at $400 \mathrm{~nm}$ by freezing the CNT motion, and again observed significant $\mathrm{O}-\mathrm{H}$ elongation, indicating the formation of $\mathrm{H}, \mathrm{OH}$, and $\mathrm{O}$ radicals; see Fig. 2(c). We thus conclude that the influence of radial expansion of the
CNT on $\mathrm{H}_{2} \mathrm{O}$ decomposition is marginal. Upon performing an extended simulation, we observed oscillation of the radius rather than breakage of the CNT. The radial expansion may be dependent on the FWHM of the laser pulse, which will be explored when performing simulations over longer timescales becomes possible.

The general question is whether there exists a preferred location of the $\mathrm{H}_{2} \mathrm{O}$ molecules for the decomposition. To address this issue, we monitored the profile of the self-consistent field potential at $800 \mathrm{~nm}$, as shown in Fig. 3(b), with the corresponding time indicated by the arrow in Fig. 3(a). The detailed profile originating from the ion potential was smeared out by extracting the self-consistent field potential without an optical E-field, and the value of the potential was averaged

(a)

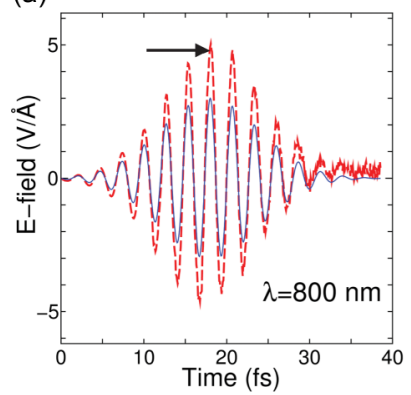

(b)

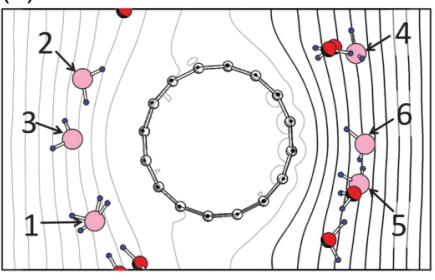

FIG. 3. (a) Time variation of applied and generated E-fields around an $(8,0)$ CNT with an optical wavelength of $800 \mathrm{~nm}$ and a maximum field intensity $\left(E_{\max }\right)$ of $3 \mathrm{~V} / \AA$. The solid (blue) line indicates the applied E-field, and the thick dashed (red) line indicates the E-field at a distance of $3.3 \AA$ from the CNT wall along the direction of optical polarization. (b) Contour lines showing the total potential change of a pristine $(8,0)$ CNT taken for the potential difference between $t=0$ and $18 \mathrm{fs}$ [see arrow in (a)] with a pulse of wavelength $800 \mathrm{~nm}$ and $E_{\max }=3 \mathrm{~V} / \AA$. The interval of the potential contour is $2.48 \mathrm{~V}$. This contour map is drawn on a plane perpendicular to the CNT axis. The initial positions of the $\mathrm{H}_{2} \mathrm{O}$ molecules are superimposed. 


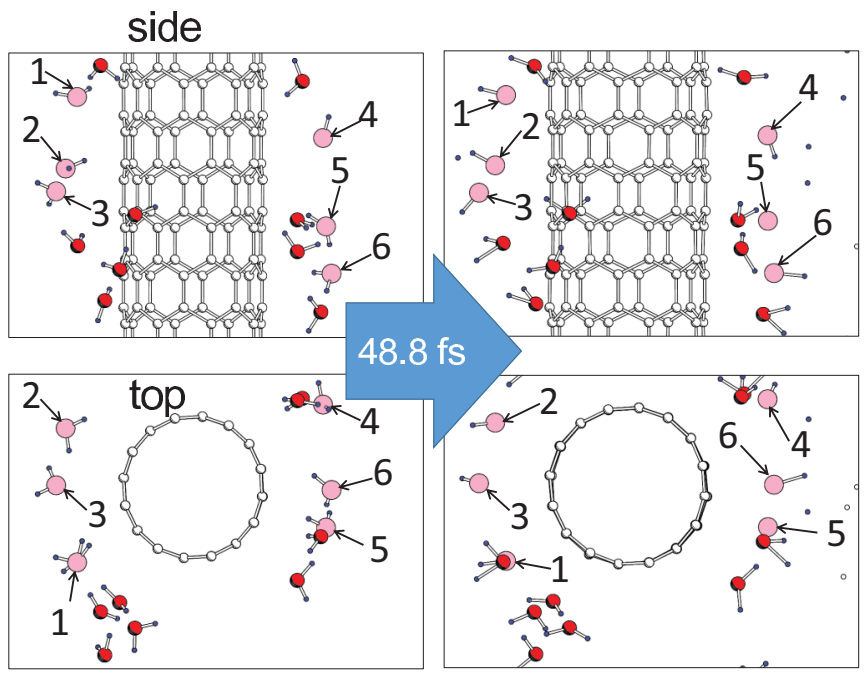

FIG. 4. Snapshot of the RT-TDDFT-MD results for the case shown in Fig. 2(a). The two left panels are at $t=0$ and the two right panels are at $t=48.8 \mathrm{fs}$. The $\mathrm{O}$ atoms of decomposed $\mathrm{H}_{2} \mathrm{O}$ molecules are depicted by larger circles with lighter shading and indicated by numbers $1-6$.

along the CNT axis. Since the intervals between the potential contour lines show the field, the optical field concentration around the CNT can be observed. By superimposing the initial positions of the $\mathrm{H}_{2} \mathrm{O}$ molecules, the molecules indicated by numbers 1-6 should be exposed to a stronger field, and these molecules indeed decomposed, as demonstrated in the snapshot of the TDDFT-MD results presented in Fig. 4. This figure shows side and top views of the system for $t=0$ and $t=48.8 \mathrm{fs}$. (The diameter of the CNT is expanded by $0.69 \AA$ at $48.8 \mathrm{fs}$, and this expansion initializes oscillation in the radial breathing mode rather than destroying the CNT.) This unit cell provides six pairs of $\mathrm{OH}$ and $\mathrm{H}$ radicals. Therefore, the amount of $\mathrm{H}$ production provides a lower value for the decomposition yield since larger numbers of $\mathrm{H}_{2} \mathrm{O}$ molecules have not yet been examined.

\section{CONCLUDING REMARKS}

In summary, the enhancement of the laser-induced decomposition of $\mathrm{H}_{2} \mathrm{O}$ in the vicinity of a CNT was demonstrated by RT-TDDFT Ehrenfest dynamics. The enhancement factor exceeds that obtained using two-dimensional sheets in our previous work [8]. The mechanisms underlying the enhancement are understood to be polarization and curvature effects of the CNT. For an applied laser pulse with an FWHM of $10 \mathrm{fs}$, the threshold power required for $\mathrm{H}_{2} \mathrm{O}$ decomposition was reduced by more than a factor of four with a wavelength of $800 \mathrm{~nm}$, and by more than a factor of seven with a wavelength of $400 \mathrm{~nm}$, compared with the case of an isolated $\mathrm{H}_{2} \mathrm{O}$ molecule. At a wavelength of $400 \mathrm{~nm}$, significant radial expansion of the CNT was observed, which turned into oscillation during extended simulations. The influence of the radial expansion on $\mathrm{H}_{2} \mathrm{O}$ decomposition was found to be negligible. Since the laser power is controlled by beam focusing, reducing the power is beneficial for allowing irradiation of a larger area and thus obtaining a higher yield of $\mathrm{H}$ atom production. In this study, we focused our attention on single-walled CNTs, but we expect that the use of multiwalled CNTs would be of future interest. Although multiwalled CNTs possess larger diameters, they should also exhibit higher polarizabilities. Electrochemical $\mathrm{H}_{2} \mathrm{O}$ decomposition, in which CNTs played a role to provide wide surface area for catalytic metals coated on CNT surfaces, was studied [33,34]. It would also be interesting to explore the effect of enhancement of electric field in an electrochemical reaction as experienced in the present work. Finally, it should be noted that nanotubes with thicker diameters with smaller band gap than the current $(8,0) \mathrm{CNT}$ could be the subject of study. In the meantime, we must note that the field enhancement would be closer to those obtained in planar graphene [8] as the diameter is increased.

\section{ACKNOWLEDGMENTS}

Calculations were performed on the supercomputing system at the Cyberscience Center of Tohoku University. Y.M acknowledges funding from JSPS KAKENHI (Grants No. JP16H00925, No. JP16H04103, No. JP16K05412, and No. JP16K05049). Y.M. also acknowledges a suggestion from $\mathrm{H}$. Ohmura regarding currently available fs laser technology. H.Z. acknowledges financial support from the National Key R\&D Program of China (Grant No. 2017YFA0303600) and the National Natural Science Foundation of China (Grant No. 11474207).

X.C. acknowledges financial support from the National Natural Science Foundation of China (Grant No. 11774248). A.R. acknowledges financial support from the JSPS Fellowship program, the European Research Council (Grant No. QSpec-NewMat ERC-2015-AdG-694097), and Grupos Consolidados (Grant No. IT578-13).
[1] K. Yuan, R. Dixon, and X. Yang, Accounts Chem. Res. 44, 369 (2011).

[2] N. Bloembergen and A. H. Zewail, J. Phys. Chem. 88, 5459 (1984).

[3] R. N. Zare, Science 279, 1875 (1998).

[4] Y. Shimotsuma, K. Hirao, P. G. Kazansky, and J. Qiu, J. Appl. Phys. Jpn. 44, 4735 (2005).

[5] Z. Lin and L. V. Zhigilei, Proc. SPIE, High-Power Laser Ablation VI 6261, 62610U (2006).

[6] S. Richter, S. Döring, A. Tünnermann, and S. Nolte, Appl. Phys. A 103, 257 (2011).
[7] A. Hu, Y. Zhou, and W. W. Duley, Open Surf. Sci. J. 3, 42 (2011).

[8] Y. Miyamoto, H. Zhang, X. Cheng, and A. Rubio, Phys. Rev. B 96, 115451 (2017).

[9] W. Lü, J. Dong, and Z.-Y. Li, Phys. Rev. B 63, 033401 (2000).

[10] A. A. Green and M. C. Hersam, Adv. Mater. 23, 2185 (2011).

[11] B. Xu, T. Kaneko, Y. Shibuta, and T. Kato, Sci. Rep. 7, 11149 (2017).

[12] J. H. Walther, R. L. Jaffe, E. M. Kotsalis, T. Werder, T. Halicioglu, and O. Koumoutsakos, Carbon 42, 1185 (2004).

[13] E. Runge and E. K. U. Gross, Phys. Rev. Lett 52, 997 (1984). 
[14] P. Ehrenfest, Z. Phys. 45, 455 (1927).

[15] J. P. Perdew and A. Zunger, Phys. Rev. B 23, 5048 (1981).

[16] J. P. Perdew, K. Burke, and M. Ernzerhof, Phys. Rev. Lett. 77, 3865 (1996).

[17] Fundamentals of Time-dependent Density Functional Theory, edited by M. A. L. Marques, N. T. Maitra, F. M. S. Nogueira, E. K. U. Gross, and A. Rubio (Springer, Berlin, 2012), Vol. 837.

[18] Y. Miyamoto, Y. Tateyama, N. Oyama, and T. Ohno, Sci. Rep. 5, 18220 (2015).

[19] M. Suzuki, J. Phys. Soc. Jpn. 61, 3015 (1992).

[20] M. Suzuki and T. Yamauchi, J. Math. Phys. 34, 4892 (1993).

[21] A. Castro, M. A. L. Marques, J. A. Alonso, G. F. Bertsch, and A. Rubio, Eur. Phys. J. D 28, 211 (2004).

[22] J. Ihm, A. Zunger, and M. L. Cohen, Phys. C: Solid State Phys. 12, 4409 (1979).

[23] N. Troullier and J. L. Martins, Phys. Rev. B 43, 1993 (1991).

[24] L. Kleinman and D. M. Bylander, Phys. Rev. Lett. 48, 1425 (1982).

[25] O. Sugino and Y. Miyamoto, Phys. Rev. B 59, 2579 (1999).

[26] O. Sugino and Y. Miyamoto, Phys. Rev. B 66, 089901(E) (2002).
[27] Y. Miyamoto and H. Zhang, Phys. Rev. B 77, 165123 (2008).

[28] The small $k$-point sampling might lead to an underestimation of the polarizability of CNT. However, the underestimation is expected to be marginal since the narrow gap of $(8,0) \mathrm{CNT}$ is at the $\Gamma$ point.

[29] B. Chimier, O. Utéza, N. Sanner, M. Sentis, T. Itina, P. Lassonde, F. Légaré, F. Vidal, and J. C. Kieffer, Phys. Rev. B 84, 094104 (2011).

[30] See Supplemental Material at http://link.aps.org/supplemental/ 10.1103/PhysRevB.99.165424 for estimation of laser field intensity as well as detailed method of the molecular dynamics.

[31] B. Rethfeld, D. S. Ivanov, M. E. Garcia, and S. I. Anisimov, J. Phys. D: Appl. Phys. 50, 193001 (2017).

[32] It is not the case according to Miyamoto [Y. Miyamoto, Eur. Phys. J. B 91, 228 (2018)] where the E-field enhancement occurs immediately after applying the external field due to an artificial effect of the periodic boundary conditions.

[33] X. Wang, H. Zhou, P. Li, and W. Shu, J. Mater. Res. 28, 927 (2013).

[34] L. Cozzarini, G. Bertolini, S. T. Šuran-Brunelli, A. Radivo, M. V. Bracamonte, C. Tavagnacco, and A. Goldoni, Int. J. Hydrogen Energy 42, 18763 (2017). 